\title{
Multiscale lensless visual position sensing by digital holography
}

\author{
Maxime Jacquot ${ }^{1}$, Miguel A. Asmad Vergara ${ }^{1,2}$, Guillaume Laurent ${ }^{1}$, Patrick Sandoz ${ }^{1}$ \\ 1. FEMTO-ST Institute, CNRS \& Univ. Bourgogne Franche-Comté, Besançon, France \\ 2. Physics Section, Department of Sciences, Pontifical Catholic University of Peru, Lima, Peru
}

Vision is a powerful contact-less control method that is widely used in many domains of applications [1-3]. However, because of physical laws describing refractive imaging systems, major parameters (magnification, lateral resolution, depth of focus, working distance, field of observation) are tied with each other in a contradictory way. An increase in magnification leads to an improvement in lateral resolution at the detriment of other parameters and vice-versa. The only ways to improve all parameters simultaneously is to reduce the imaging wavelength or to increase the refractive index but, in practice, such solutions present several highly limiting constraints.

This paper presents how the use of digital holography (DH) as a lensless vision system applied to in-plane position sensing provides some way to make the measurement performances more independent of each other [4]. In $\mathrm{DH}$, the working distance, numerically tuned at the image reconstitution stage, is virtually unlimited except by setup specifications such as the laser source coherence length and the solid state image sensor size. An extended pseudoperiodic pattern is used as a target. The latter makes the allowed displacement range independent of the vision-system field-of-observation whereas periodicity allows phase measurements leading to a high degree of subpixel interpolation. In this way, major limiting constraints of refractive imaging systems are released, thus offering exceptional multiscale measurement capabilities, despite the same physical laws still apply. In practice, we demonstrated in-plane position measurement of a moving target with a 50nm lateral resolution, $10^{-3} \mathrm{deg}$. angular resolution, an allowed displacement range of $20 \mathrm{~mm} / 2 \pi$, over a $15 \mathrm{~cm}$ range of working distances. The depth of focus (DoF) is similar to that obtained with a refractive imaging system with the same numerical aperture but, in $\mathrm{DH}$, it can be numerically centred on the current working distance, thus releasing DoF limitations. This level of performances shows that, at the cost of an interferometric setup and numerical image reconstruction (recorded hologram Fig. 1(a), reconstructed image Fig. 1(b)), DH constitutes an outstandingly tuneable imaging system providing unique capabilities for position sensing applications.

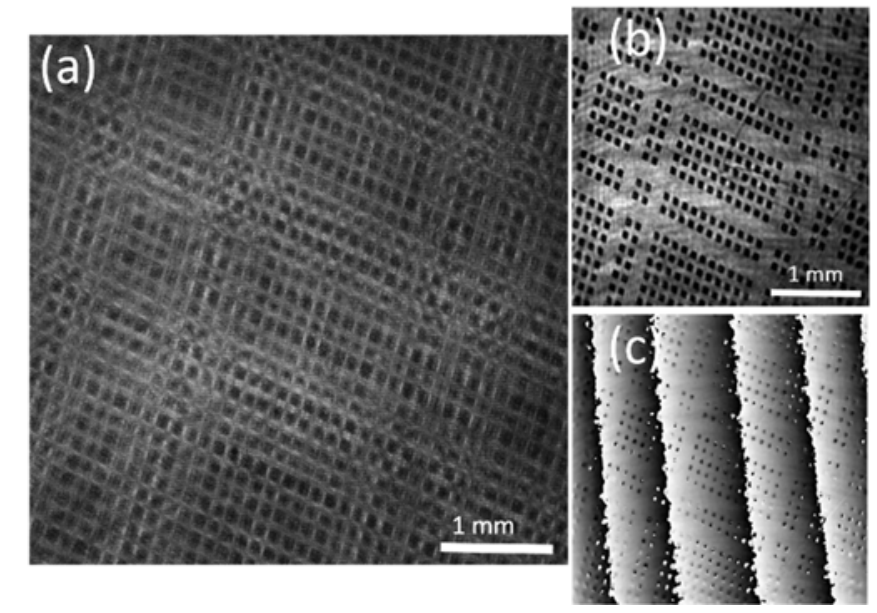

Fig. 1 (a) Experimental off-axis digital hologram recorded. (b) Amplitude image in focus reconstructed numerically from the hologram and, (c) phase image extracted from the hologram with the tilted plane representative of the out-of-axis angle.

Beyond these imaging capabilities, DH is an interferometric technique that provides phase. The latter can be used for supplementary out-of-plane sensing with an excellent resolution. The axial direction $\mathrm{Z}$ is subject to phase ambiguities that prevent absolute measurements but allows the reconstruction of relative position if the sampling rate is sufficiently high in regard to the $\mathrm{z}$-displacement speed. Out-of-plane angles are reconstructed in an absolute way with a high resolution from the mean phase plane orientation as represented in Fig. 1(c).

\section{References}

[1] R. Szeliski, Computer vision: algorithms and applications. (Springer Science \& Business Media, 2010).

[2] J. Galeano, P. Sandoz, E. Gaiffe, S. Launay, L. Robert, M. Jacquot, F. Hirchaud, J.L. Prétet, C. Mougin, « Positionreferenced microscopy for live cell culture monitoring. " Biomedical optics express, 2(5), 1307-1318 (2011).

[3] N. Tan, C. Clévy, G.J. Laurent, P. Sandoz, N. Chaillet «Accuracy quantification and improvement of serial micropositioning robots for in-plane motions. IEEE Transactions on robotics, »31(6), 1497-1507 (2015).

[4] P. Sandoz, M. Jacquot, "Lensless vision system for in-plane positioning of a patterned plate with subpixel resolution. 》 J. Opt. Soc. Am. A 28, 2494-2500 (2011). 